Revista Brasileira de Agricultura Irrigada v.12, no .2, p. $2487-2499,2018$

ISSN 1982-7679 (On-line)

Fortaleza, CE, INOVAGRI - http://www.inovagri.org.br

DOI: 10.7127/rbai.v12n200765

Protocolo 765.18 - 18/09/2017 Aprovado em 17/01/2018

\title{
MENSURAÇÃO FENOLÓGICA E FOTOGRAMÉTRICA FOLIAR COM LASERS E CÂMARA DIGITAL
}

\author{
Gervásio Fernando Alves Rios ${ }^{1}$, Fabiana Carmanini Ribeiro² ${ }^{2}$ José Alves da Mata ${ }^{3}$, João José \\ da Silva Júnior ${ }^{4}$, Gabriela Souza Oliveira ${ }^{5}$
}

\begin{abstract}
RESUMO
O objetivo deste trabalho foi apresentar uma metodologia para determinação da escala fotogramétrica de imagens obtidas em câmara digital comum sem a necessidade de referência fixa de escala no espaço objeto; a metodologia foi aplicada em situações do meio agrícola em que o objeto a ser mensurado compõe-se de superfícies planas e; nesse contexto, visa estimar a cobertura do solo, biomassa, estádios fenológicos, monitoramento da capacidade produtiva, consumo hídrico, intensidade do ataque de pragas/doenças, previsão de safra, entre outros aspectos ligados à agricultura e trabalhos de pesquisas. Esse trabalho foi desenvolvido utilizando-se de um protótipo laser de escala (E, modelo m.L1), de vários apontadores lasers, acoplado a uma câmera digital marca Olympus (mod. FE340); foram realizados vários ensaios específicos para estimativa, calibração e validação de desempenho dos parâmetros ( $\beta$, Xo, d') do protótipo (m.L1) e de modelos funcionais vinculados (m.1, 2 e 3), dependentes da distância (X) da imagem (m.1), do referencial homógrafo (d') de um (m.2) ou mais pares de lasers (m.3) em relação ao padrão de grade (m.G). Concluiu-se que os parâmetros do protótipo e modelos tiveram acurácia e desempenho satisfatórios, não recomendado sua utilização a uma distância da câmera inferior a $0,4 \mathrm{~m}$.
\end{abstract}

Palavras-chave: Área foliar, Experimentação Agrícola, Fenometria.

\section{PHOTOGRAMMETRIC AND LEAF PHENOLOGY MEASUREMENT WITH LASERS AND DIGITAL CAMERA}

\footnotetext{
${ }^{1}$ Eng. Agrônomo, Professor Adjunto, Universidade de Brasília FAV/UnB, e-mail: gervasiorios@unb.br

${ }^{2}$ Eng. Agrônoma, Professora Adjunta, Universidade de Brasília - FAV/UnB, e-mail: facarmanini@ unb.br

${ }^{3}$ Arquiteto e Urbanista, Centro Universitário Metodista Izabela Hendrix,INTERTECHNE, email:jam@hendrix.br

${ }^{4}$ Eng. Agrônomo, Professor Adjunto, Universidade de Brasília FAV/UnB, e-mail: jjsjunior@unb.br

${ }^{5}$ Graduanda de Arquitetura e Urbanismo, Universidade de Brasília - FAU/UnB, e-mail: gabioliveira.arq@gmail.com
} 


\begin{abstract}
This experiment was aimed atpresent a methodology for the determination of the photogrammetric scale of images obtained in common digital camera without the need for fixed reference of scale in the object space; the methodology has been applied in situations of agricultural environment in which the object to be measured is composed of flat surfaces and; in this context, aims at estimating the soil cover, biomass, phenological stadiums, monitoring of production capacity, water consumption, intensity of attack by pests/diseases, crop forecast, among other aspects relating to agriculture and research work. This study was conducted using a laser scale prototype (E, m.L1 model), of several lasers pointers, coupled to an Olympus digital camera (model FE340); several specific assays were performed for estimation, calibration and validation performance parameters $\left(\beta\right.$, Xo, $\left.d^{\prime}\right)$ of the prototype (m.L1) and related functional models (m.1, 2 and 3) dependent on the distance $(\mathrm{X})$ of the image $(\mathrm{m} .1)$, of the referential homograph (d') of a (m.2) or more pairs of lasers (m.3) compared to the standard model grid (m.G). It was concluded that the parameters of the protoype and models have had accuracy and satisfactory performance, not recommending their use at a distance of less than $0.4 \mathrm{~m}$ camera.
\end{abstract}

Keywords: Agricultural experimentation, Fenometria, Leaf area.

\section{INTRODUÇÃO}

A discretização da imagem, aumento da capacidade de armazenamento e processamento da informação, popularização das câmeras digitais e equipamentos eletrônicos vêm promovendo avanços e possibilidades nunca antes experimentados em diferentes campos do conhecimento humano. No campo agrícola a fotogrametria e o sensoriamento remoto se destacam como técnicas e meios de quantificação e estimativa da cobertura do solo, biomassa, consumo de água entre outros aspectos ligados à agricultura e trabalhos de pesquisas. Nesse contexto, um protótipo acoplado à câmera fotográfica digital comum e modelos vinculados para determinação da escala fotogramétrica de imagens foram desenvolvidos tendo como motivação a mensuração acurada e periódica de plantas (ou suas partes: dimensões foliar, projeção da copa, altura, etc.) ao longo de seu ciclo vegetativo, de forma simples, ágil, por amostragem destrutiva ou não da planta, independentemente; com reduzida mão-deobra e; tendo em vista um baixo custo, praticidade e acurácia de medidas a serem processadas e analisadas em trabalhos de pesquisas e avaliações de cultivo no campo.
Essa motivação teve origem na necessidade de mensuração de variáveis fenométricas e biométricas das plantas, sob distintas condições experimentais de campo e finalidades relacionadas: às estimativas de consumo de água (evapotranspiração); capacidade fotossintética das plantas (produção de biomassa, taxa de ocupação/cobertura do solo, estádios e escala fenológica do ciclo da cultura) e; às análises do ataque de pragas e doenças (intensidade/ severidade, nível de dano econômico e manejo agrometeorológico); assim como, pelas dificuldades de obtenção dessas medidas no campo, em decorrência, dentre outros fatores, do elevado custo operacional e, ou uso de instrumentos quase sempre caros e importados (a exemplo do LI-Cor 3100c Portable Leaf Area Meter, LI-Cor 3000c, LAI-2200, CI-202 Leaf Area Meter, entre outros). Esse elevado custo é uma das razões pelas quais, salvaguardadas as exceções, muitos experimentos e trabalhos de pesquisa básica poderiam ser mais bem explorados e alcançar resultados mais avançados.

O levantamento quantitativo e periódico desses parâmetros é fundamental, pois integram importantes variáveis de estudos da fisiologia, fenologia, fitopatometria, fitotecnia, agrometeorologia, sensoriamento remoto e 
servem para fins de estimativa, simulação, previsão em modelos de análise quantitativa do crescimento/desenvolvimento vegetativo; grau de ataque de pragas/doenças; às estimativas da evapotranspiração e manejo da irrigação; componentes de produção das plantas e produtividade (ou safra). Em síntese, destacase a importância de se mensurar/estimar a área foliar (AF) ou o índice de área foliar (IAF = $\mathrm{AF} / \mathrm{At}$ ) definido pela razão entre a área foliar média da planta e, ou comunidade vegetal (AF) e sua área útil total explorável (At) ao longo do ciclo; visto que, esse é o principal parâmetro biométrico das plantas, expressando o estado de crescimento e desenvolvimento do vegetal e que está estritamente correlacionado aos três principais fatores da produção vegetal: fotossíntese, fitossanidade e consumo de água (evapotranspiração).

Nesse sentido, vários são os trabalhos que devem e que foram consultados para o embasamento e desenvolvimento desses dispositivos, destacando-se alguns deles relacionados: a) ao estudo de imagens digitais, fotogrametria e uso de softwares (AFSoft; SIARCS; ImajeJ (R) para o processamento e análise de imagens (BRITO; COELHO FILHO, 2007; BERVEGLIERI et al., 2015; PAVAN; SANTOS, 2015); b) à mensuração de área foliar (SEVERINO et al., 2004; BOSCO et al., 2012; KAUR et al., 2014; LIMA et al., 2014; SACHET et al., 2015); c) à estimativa do Índice de Área Foliar (BENINCASA, 2003; MARCON et al., 2011); d) à estimativa do Índice de Área Foliar e análise quantitativa do crescimento/desenvolvimento (BENINCASA, 2003; PEIXOTO et al., 2011); e) ao uso de modelos de simulação do crescimento (Aquacrop, entre outros) e Escala fenológica (TOUMIA et al., 2016); f) à estimativa da área de projeção da copa, fator de cobertura e avaliação da cobertura do solo (MARTINS NETO et al., 2013); g) à estimativa do ataque de pragas/doenças de plantas (JORGE; SILVA, 2009; SANTOS et al., 2010) e; h) outros relacionados à importância de estimativa da área foliar da planta, sua relação com a evapotranspiração, mecanismos da relação fonte-dreno e outras aplicações (ALLEN et al., 1998; RIOS et al., 2013).

Nesse contexto, propôs-se com este estudo, alcançar os seguintes objetivos: a) desenvolver uma metodologia cujo dispositivo laser acoplado à câmera digital comum tenha por finalidade a determinação da escala fotogramétrica de imagens obtidas de objetosalvo de tamanhos, formas, amostragem (indestrutiva ou não) e situações de ambientes variados, ligados à produção e, ou pesquisas de experimentação agrícola e; b) fazer estimativa, calibração e validação de desempenho dos parâmetros do modelo protótipo e modelos funcionais associados, em relação a um modelo padrão, dependentes da distância da imagem-objeto, do referencial homógrafo de um ou mais pares de lasers.

\section{MATERIAL E MÉTODOS}

O desenvolvimento de dispositivo laser, modelos funcionais e experimentos calibração tiveram como motivação e pré-requisitos os conhecimentos empreendidos por iniciativa própria e durante os cursos de graduação e pósgraduação; a participação e realização de experimentos de pesquisa; as análises e elaboração de artigos, resumos e projetos de pedido de patente.

\section{Desenvolvimento do dispositivo laser}

O dispositivo laser funciona acoplado a uma câmera fotográfica digital ou analógica comum para auxiliar na determinação da escala da fotografia digitalizada nas mais variadas situações de tomada. Esse dispositivo projeta na cena imageada um padrão de luz estruturada (na forma de gride ou referência de escala), composto de feixes lasers projetados na cena e cujas medidas, em pixel, juntamente com as medidas do padrão de referência conhecido (em metro), são processadas e determinam a escala da imagem (em pixel/metro), conforme ilustração da Figura 1. 
a)

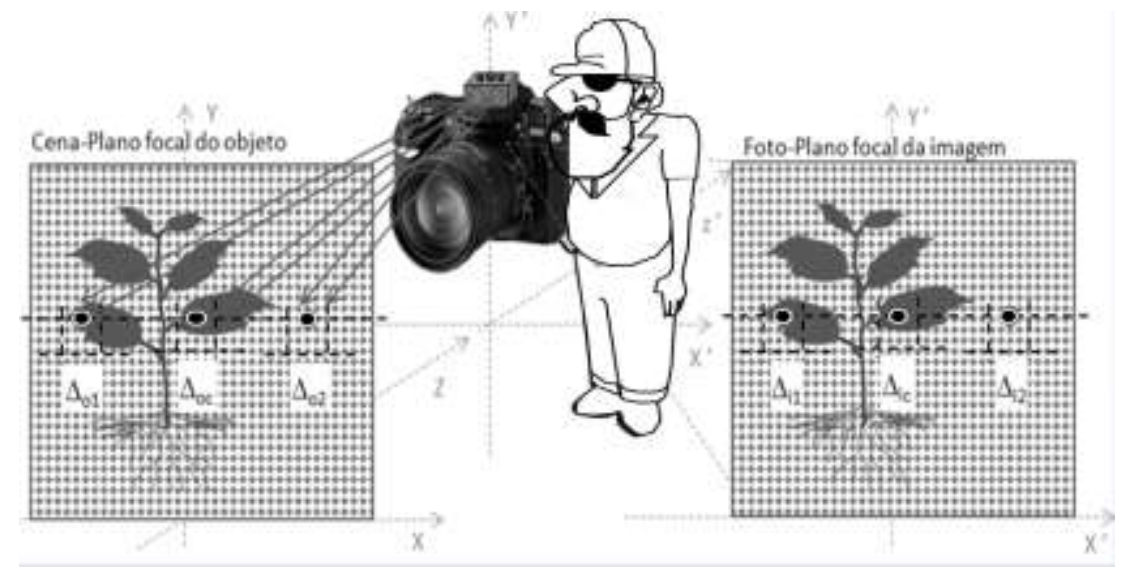

b)

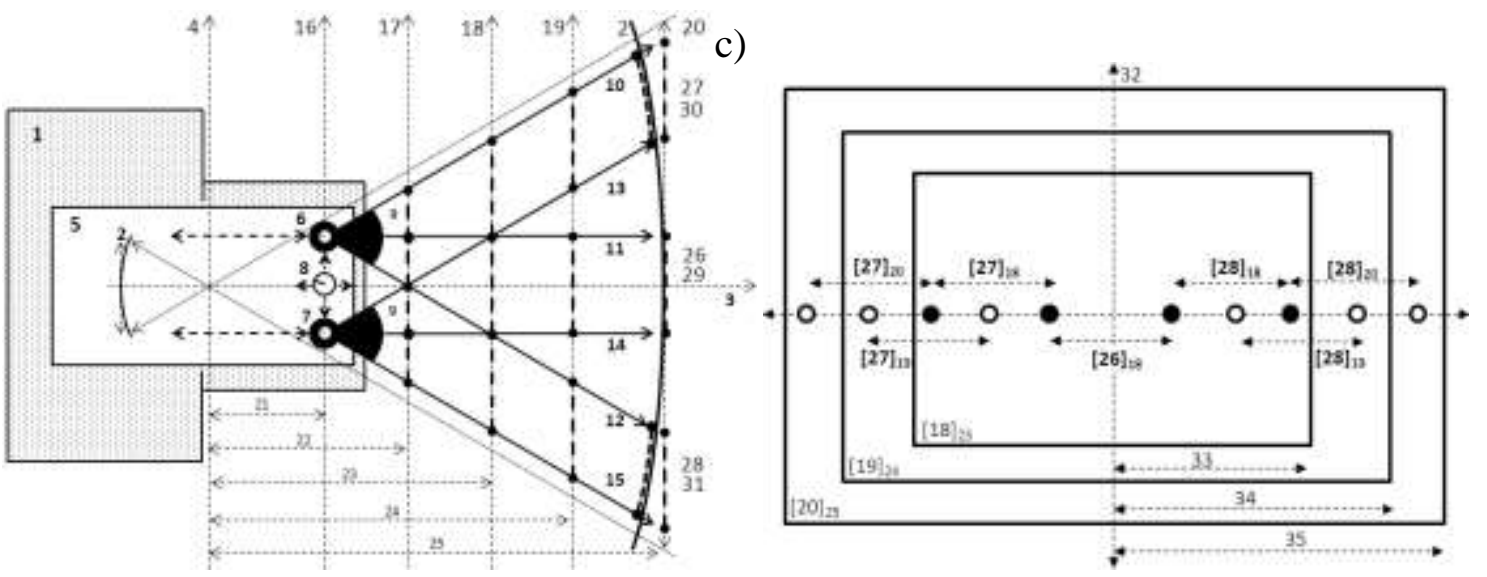

Fonte: elaborado pelo autor.

Figura 1. a) ilustração do funcionamento do invento acoplado à câmara fotográfica; b) vista superior do funcionamento e componentes do invento (5) e suas fontes de projeção laser (6 e 7) acoplado à câmara fotográfica (1), projetando o padrão de referência de escala (26, 27 e 28, em m) no plano focal do objeto (17, $18,19, . ., 20)$ com seus correspondentes virtuais $(29,30$ e 31 , em pixel) variáveis com a profundidade (21, 22, $23,24 \ldots, 25$, em $\mathrm{m}$ ) e a superfície de distorção da lente (2, parábola tangente ao plano focal da imagem e, também por convenção, ao plano focal do objeto 20); c) ilustração da sequência de imagens fotografadas nos planos 18,19 e 20, às distâncias $23<24<25\left([18]_{23}\right.$, [19] ${ }_{24}$ e [20] $\left.{ }_{25}\right)$; respectivas referências de escala laser no plano 18 ([26] $]_{18},[27]_{18}$ e $\left.[28]_{18}\right) ; 19$ ([26 $]_{19},[27]_{19}$ e $[28]_{19}$ e $20\left([26]_{20},[27]_{20}\right.$ e $\left.[28]_{20}\right)$; e suas respectivas proporções $(\mathrm{em} \mathrm{m} / \mathrm{m})$ em relação aos seguimentos 33, 34 e 35 de cada plano 18, 19 e 20.

Com o uso de feixes lasers projetados na forma pontual, duas configurações ou modelos são considerados: um em que de cada fonte apenas um feixe pontual é projetado (m.L1), e outro, em que três feixes de cada fonte são projetados (m.L3). Na configuração mais simples (m.L1), ambos os pontos de projeção foram orientados na horizontal e em paralelo entre si (e com o eixo ótico de profundidade da câmera) para formar dois pontos luminosos nos planos do objeto imageado a um dada distância. A outra configuração complementar e semelhante (m.L3), porém, de maior abrangência da parte periférica da cena imageada (Figura 1b) é obtida adicionando em cada fonte emissora e ao plano de projeção mais dois feixes pontuais a Laser, um de cada lado da fonte (externo e interno). Em cada fonte é formado um ângulo de abertura conhecido em relação ao feixe central, tanto pelo seu lado externo quanto interno, ambos de modo simétrico e idêntico. Esse ângulo será regulável em relação ao feixe central ou eixo ótico por ajustadores de posição situados sobre as fontes na plataforma, de tal forma a manterem paralelo o feixe externo de uma fonte com o interno da outra, e vice-versa. Esse ângulo pode ser no máximo a metade do ângulo de abertura da câmera. Assim, entre cada par de feixes pontuais paralelos (um de cada fonte), complanares e projetados horizontalmente, formam-se seguimentos de 
reta ao centro e nas laterais da cena imageada. Esses segmentos projetados possuem dimensões reais fixas e determinadas que, após processamento computacional, servem de base para obtenção homográfica da escala da imagem e, ou sua relação com as dimensões da imagem, Figura 1, independente da distância de registro da cena.

A projeção de feixes lasers na forma laminar, ou seja, linha formada por inúmeros pontos laser contíguos (a semelhança dos leitores de códigos de barra), seguem as mesmas características da projeção na forma pontual, podendo ter disposição de dois ou mais fontes emissoras dos feixes, podendo também ser dispostas na vertical, isto é, uma acima da outra na cena imageada, com projeções de duas ou mais linhas lasers horizontais paralelas.

\section{Modelos de medida pelo protótipo-laser}

$\mathrm{Na}$ Figura 2 abaixo é apresentado o protótipo dispositivo laser (m.L1), calibrado e usado em trabalhos de pesquisa, acoplando a uma câmera digital da marca Olympus Imaging (mod. FE340). Ressalta-se que apensar da potencialidade de uso de mais de um pares de lasers (m.L3) o protótipo desse modelo ainda não foi construído.
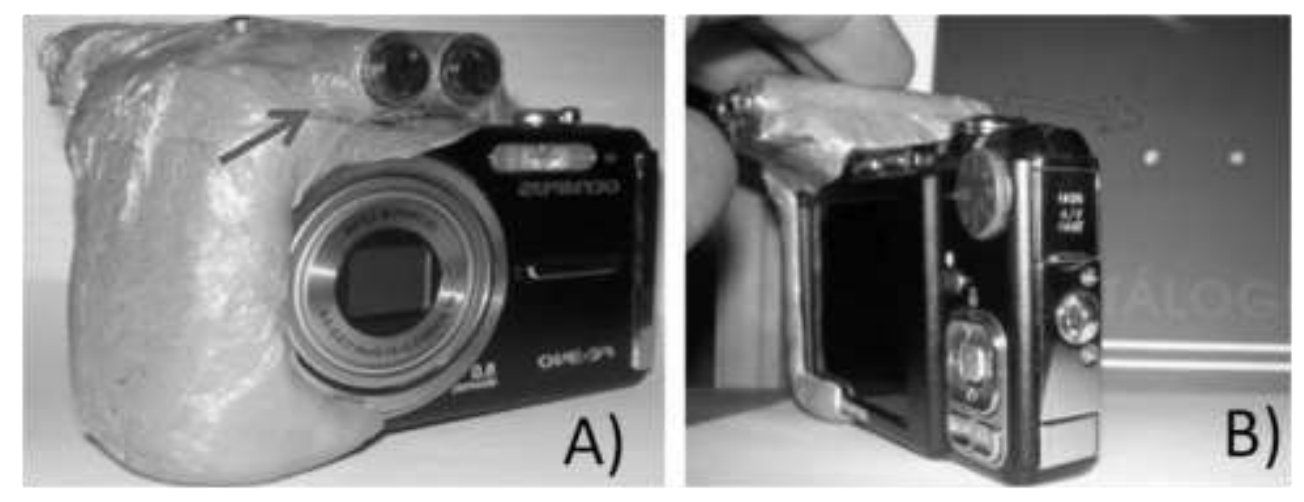

Fonte: elaborado pelo autor.

Figura 2. A) ilustração de um protótipo m.L1 indicado com setas as fontes do par de lasers e B) suas projeções (pontos claros) na parede.

Os métodos de medida do protótipo laser se referem aos modelos de determinação da escala da imagem (E, em pixel/cm) atendidos alguns pressupostos e observações comuns: i) os modelos destinam-se às estimativas das dimensões de objetos planos no plano objetoimagem; ii) as imagens (fotos) devem ser de baixa variação da escala no plano imagem, o que requer máquinas com lentes de baixa distorção e configuração no modo padrão (normal), e, ou ortorretificação computacional da imagem, sem necessidade de calibração dos parâmetros internos e externos da câmera digital; iii) o dispositivo laser deve ser calibrado e permitir que $o$ par de lasers de preferência (projetado no centro da cena, quando constituído de um par apenas) permaneça perfeitamente paralelos (com sua projeção referencial de tamanho fixo, d), ou o mais paralelo possível (com seu referencial pouco variável, d'), independente da distância do plano objetoimagem e; iv) a determinação acurada da escala pelo protótipo e modelos associados exige que o protótipo laser seja calibrado, validado e os lasers (quando formado de apenas um par) sejam projetados de preferência no centro da cena.

Com base nessas observações foram analisados cinco modelos para a obtenção da escala da imagem (E, em pixel $/ \mathrm{cm}$ ) pelo protótipo laser, três deles (m.1, m.2 e m.3) foram baseados na estimativa de uma função específica de escala. Esses modelos são descritos a seguir.

\section{Modelos diretos de obtenção de escala da imagem (m.L1 e m.G)}


Os modelos m.L1 e m.G basearam-se na estimativa direta da escala com base apenas na medida homográfica da imagem (yi ou y'i, em pixel) correspondente à projeção fixa do referencial de medida conhecida (d ou d', em $\mathrm{cm}$ ) do par de lasers (m.L1); ou conforme o uso de uma grade de $30 \times 30 \mathrm{~cm}$ (m.G), circunscrita às plantas de alface imageadas a uma altura fixa de $100 \mathrm{~cm}$, adotado nesse estudo, como modelo padrão de referência. A estimativa da escala no m.L1 pode ser diretamente calculada caso o par de lasers forem perfeitamente paralelos. Caso não existam condições práticas para construir o dispositivo laser com seu par de feixes perfeitamente paralelos, e sim como ocorreu nesse trabalho, condições suficientes para torná-los o mais paralelo possível (com pequena divergência entre eles), e nesse caso, a escala foi obtida pela razão entre a homógrafa (y'i, em pixel) e seu correspondente corrigido $\left(E=y^{\prime} \mathrm{i} / \mathrm{d}^{\prime}\right.$, pixel $\left./ \mathrm{cm}\right)$. Dessa forma, para esse caso existe a necessidade de fazer calibração e validação de alguns parâmetros, via teste e ensaios específicos, como foi feito, ou fazer uso de modelos funcionais de escala que permitem a obtenção imediata de escala das imagens e seu processamento, conforme descrito adiante.

\section{Modelo funcional de obtenção de escala com a distância (m.1)}

O modelo m.1 é uma função de escala da imagem $(E=f(X)$, em pixel $/ \mathrm{cm})$, variável com a distância de tomada do plano do objetoimagem $(\mathrm{X}, \mathrm{em} \mathrm{cm})$ para um protótipo-laser específico, e que pode ser ajustada por regressão mediante um experimento simples de calibração e levantamento de medida de pares ordenados $\left(X_{i} ; E_{i}=f\left(X_{i}\right)\right)$, tal que seja $E_{i}$ $=\mathrm{y}_{\mathrm{i}} / \mathrm{d}$ ' para o par de lasers pouco paralelos ou $\mathrm{E}_{\mathrm{i}}=\mathrm{y}_{\mathrm{i}} / \mathrm{d}$ para lasers perfeitamente paralelos, conforme o modelo m.L1. A aplicação desse modelo se justifica para os casos em que se conhece ou se pode medir com facilidade a distância de tomada da fotografia (Xi) e uma função bem ajustada. Uma das alternativas úteis de uso desse modelo é para a estimativa da escala de projeção da copa de uma planta a partir das medidas de altura de uma régua graduada $(\mathrm{Xr})$ colocada no plano de projeção da copa ao solo (Figura 3).

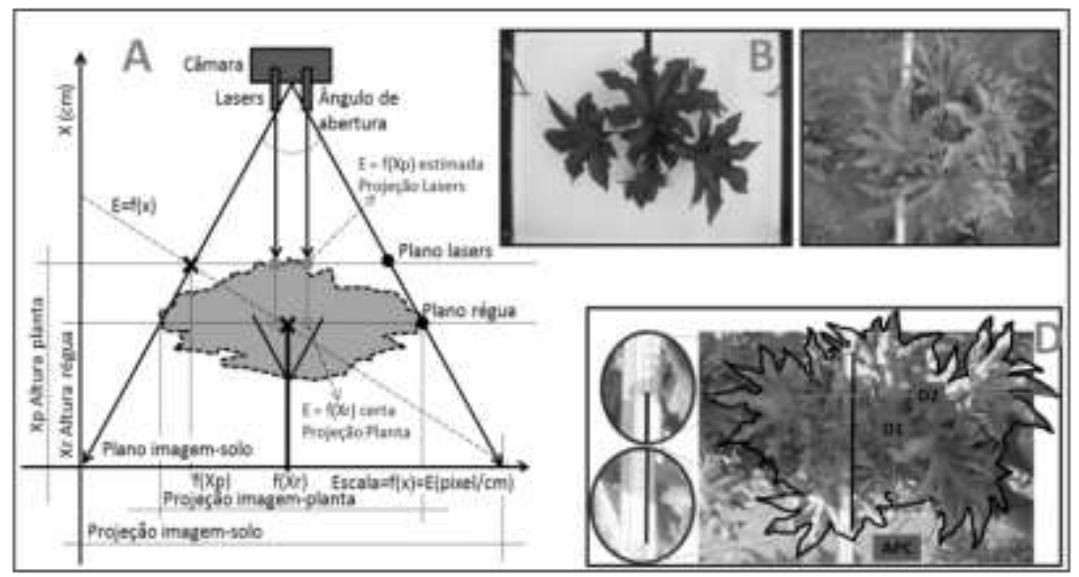

Fonte: elaborado pelo autor.

Figura 3. Ilustração de uso (A), aplicação e ajuste da função de escala $(E=f(x))$ com a distância $(X)$ para a estimativa de dimensões (D1 e D2) e área de projeção da copa da planta (APC) no plano da imagem com auxilio da régua graduada $(B, C$ e $D)$.

\section{Modelo funcional de obtenção de escala com a projeção (m.2)}

O modelo m.2 é uma função de escala da imagem $(E=f(y)$, em pixel/cm), variável com o tamanho da projeção homográfica (yi ou y’i, em pixel) do referencial fixo (d ou d' em $\mathrm{cm}$ ) para um protótipo-laser específico, independente da distância Xi de tomada da cena imageada, e que pode ser ajustada por regressão mediante um experimento de simples calibração e levantamento de medida 
de pares ordenados $\left(y^{\prime} ;\right.$; Ei $\left.=f\left(y^{\prime}{ }_{i}\right)\right)$, tal que $E_{i}$ $=\mathrm{y}_{\mathrm{i}} / \mathrm{d}$ ' para o par de lasers pouco paralelos ou $\mathrm{Ei}=\mathrm{y}_{\mathrm{i}} / \mathrm{d}$ para lasers perfeitamente paralelos, conforme o caso, para o protótipo modelo m.L1. A aplicação desse modelo se justifica pela praticidade de se estimar a escala de determinada imagem digital pelo dispositivo protótipo-laser (m.L1), sob as mesmas configurações em que foi ajustado o modelo, independentemente de Xi.

\section{Modelo funcional de obtenção de escala média com as projeções (m.3)}

O modelo m.3, semelhante ao modelo $\mathrm{m} .2$, se distingue desse por ser uma função de escala média da imagem $(\mathrm{E}=\mathrm{f}(\mathrm{y})$, em $\mathrm{pixel} / \mathrm{cm}$ ) para um protótipo-laser específico, particularmente de três pares de lasers (m.L3) conforme Figura 1. Nesse caso, basta obter diretamente da imagem o tamanho médio da homógrafa (yi ou y'i, em pixel) correspondente à média das projeções dos pares de lasers (di ou d'i, em cm) e entrar com esse valor na função para proceder com as conversões das medidas digitais de interesse para o sistema métrico. A estimativa da escala média por esse modelo foi obtida dos ensaios de calibração realizados com uma régua graduada (em $\mathrm{mm}$ ) fixada numa parede e no centro da cena imageada. No centro da régua foi projetado o par de lasers central do protótipo (d'i) de medidas homógrafas desse referencial (y'i) e de medidas de $10 \mathrm{~cm}$ nas partes marginal (y'i3) e intermediária (y'i2) da régua e, ou imagem obtida; simulando assim,

a)

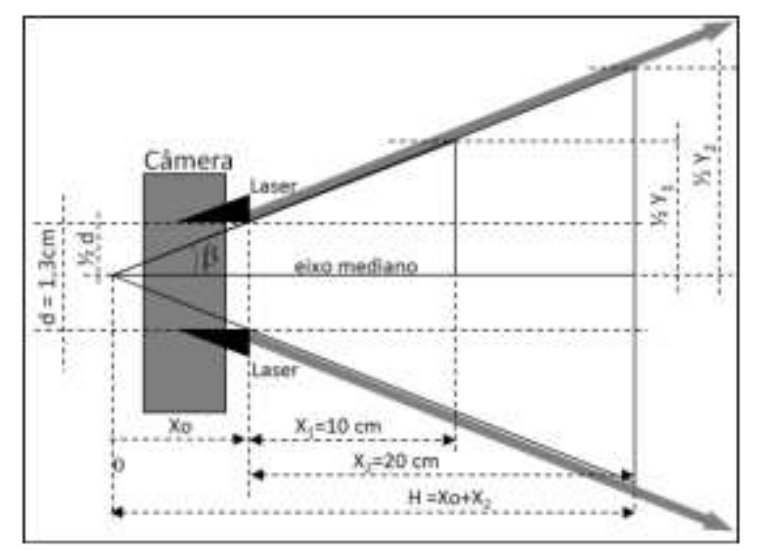

Fonte: elaborado pelo autor. as referências de mais dois pares de lasers projetados na periferia da imagem A aplicação desse modelo se justifica pela maior representatividade possível da escala média no plano da imagem (com freqüentes distorções de suas margens em razão da lente) e pela praticidade de estimá-la pelo dispositivo protótipo-laser sob as mesmas configurações em que foi ajustado o modelo. Adicionalmente o uso desse dispositivo com pares de lasers distribuídos por toda a extensão da cena e, ou imagem se justifica em aplicações específicas que requeiram maior rigor nas medidas, considerando-se a projeção de referência e sua homógrafa como um modelo matricial ([yi] x [di]), podendo inclusive ser utilizado para ortorretificação de imagem e método de calibração dos fatores internos e externos de câmeras digitais.

\section{Calibração do protótipo e estimativa de seus parâmetros}

A calibração do protótipo foi necessária em razão do uso de lasers comuns de fabricação grosseira e da disponibilidade de instrumentos adequados para se estabelecerem fixos o paralelismo entre par de lasers ou a medida do seguimento (d) formado entre os seus centros de projeção em todas as distâncias testadas a partir da cena inicial (Xo), a cada 10 $\mathrm{cm}$ entre o projetor laser e o objeto fotografado $(\mathrm{X} 1, \mathrm{X} 2, \ldots, \mathrm{Xi}, \mathrm{em} \mathrm{cm})$ até o máximo de 600 $\mathrm{cm}$, considerando o eixo mediano da projeção laser colinear ao eixo ótico da câmera, Figura 4 (a e b).

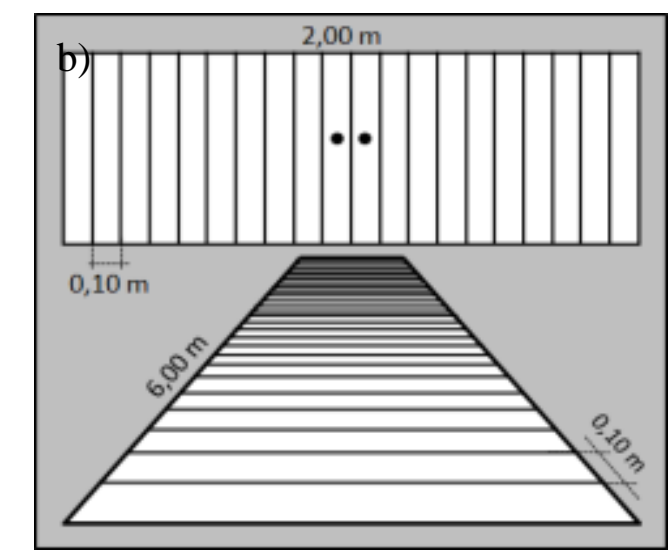

Figura 4. a) ilustração geométrica do desvio de projeção paralela do par de lasers caracterizado pelo semi- 
ângulo beta $(\beta)$ entre os lasers e o eixo mediano, distância inicial entre o início da projeção do par de lasers e o vértice entre eles $(\mathrm{Xo})$; b) Ilustração da forma de calibração experimental do paralelismo entre as projeções lasers, em perspectiva.

Esse procedimento visou determinar: a) o semi-ângulo beta médio $(\beta)$; b) a distância inicial (Xo) formada entre o seguimento inicial $(\mathrm{d}=1,3 \mathrm{~cm})$ e o vértice da projeção dos lasers e; c) o seguimento estimado (d') para todas as distâncias de tomada da foto $(\mathrm{X} 1, \mathrm{X} 2, \ldots, \mathrm{Xi})$ a partir do vértice $\mathrm{H}(\mathrm{Xo}+\mathrm{X} 1, \mathrm{Xo}+\mathrm{X} 2, \ldots$, $\mathrm{Xo}+\mathrm{Xf}$ ), conforme ilustrado na Figura 4(a e b), baseando-se nas respectivas Equações 1, 2, 3 e suas variantes (1a, 1b, 3a e 3b).

$$
\begin{gathered}
\bar{\beta}=\tan ^{-1}(\overline{\tan \beta}) \\
\overline{\tan \beta}=1 / n \cdot \sum \tan \beta_{i} \equiv \operatorname{Média}\left(\tan \beta_{i}\right) \\
\tan \beta_{i}=\frac{1 / 2 \cdot\left(\mathrm{Y}_{i+1}-\mathrm{Y}_{i}\right)}{\left(\mathrm{X}_{i+1}-\mathrm{X}_{i}\right)} \quad(1 \mathrm{~b}) \\
\mathrm{X}_{o}=\frac{1 / 2 \cdot \mathrm{d}}{\tan \bar{\beta}} \quad(2) \\
d^{\prime}=2 \mathrm{H} \cdot \tan \bar{\beta} \equiv \mathrm{H} \cdot \tan \bar{\alpha} \\
\mathrm{H}=\mathrm{X}_{o}+\mathrm{X}_{f} \\
\bar{\alpha}=2 \bar{\beta}
\end{gathered}
$$

em que,

$\beta$ é o semi-ângulo beta médio estimado pelo inverso (arco-tangente) da tangente média $\left(\tan ^{-1}(\overline{\tan \beta})\right)$ dos valores tangentes ao feixe laser a cada $10 \mathrm{~cm}$ de leituras tomadas às distâncias $\mathrm{Xi} \quad(\tan (\beta \mathrm{i})) ; \mathrm{Y}_{\mathrm{i}+1}$ e $\mathrm{Y}_{\mathrm{i}}$ são as respectivas medidas reais da projeção $(\mathrm{em} \mathrm{cm})$ do seguimento d' $(\mathrm{em} \mathrm{cm})$ tomado da imagem a cada $X_{i+1}$ e $X_{i}(e m c m)$, respectivamente; d' é o seguimento de projeção do seguimento $d$ (formado pelos centros de projeção inicial do par de lasers) e estimado para cada distância de tomada da foto a partir da distância inicial de projeção até o vértice entre os lasers (Xo) ou nas demais distâncias posteriores a partir do vértice $\mathrm{H}(\mathrm{Xo}+\mathrm{X} 1, \mathrm{Xo}+\mathrm{X} 2, \ldots, \mathrm{Xo}+\mathrm{Xf}, \mathrm{em}$ $\mathrm{cm})$; Xi é a medida de distância $(\mathrm{cm})$ entre a projeção inicial do par de lasers (Xo) e o objeto fotografado a cada $10 \mathrm{~cm}$, tomada até $600 \mathrm{~cm}$ de Xo; H é a distância estimada entre o vértice e a projeção do par de lasers (d') sobre o objeto fotografado a cada $10 \mathrm{~cm}(\mathrm{em} \mathrm{cm}) ; \mathrm{Xf}$ é a medida de distância Xi $(\mathrm{em} \mathrm{cm})$ de tomada das fotos do experimento da alface, no caso, feitas a $100 \mathrm{~cm}$ de Xo; $\beta$ é semi-ângulo de $\alpha$ formado no vértice entre os feixes lasers de cada par (em rad). Na estimativa de beta médio ( $\bar{\beta}$ ) foram excluídos do cálculo valores extrapolados (outliers) de tangentes $(\tan (\beta \mathrm{i}))$ tomadas a cada $10 \mathrm{~cm}$ de distâncias $(\Delta \mathrm{Xi})$.

\section{Validação e desempenho dos modelos}

A validação e avaliação do desempenho foram feitas para os modelos m.1, m.2, m.3 e m.L1 assumindo como padrão o modelo do uso da grade m.G. As funções dos modelos m.1, m.2 e m.3 foram ajustadas por regressão dos dados obtidos do experimento de calibração $\left(\mathrm{X}_{\mathrm{i}}\right.$; $\mathrm{y}_{\mathrm{i}}\left(\mathrm{d}^{\prime}{ }_{\mathrm{i}}\right)$ e d'i, $\mathrm{y}_{\mathrm{i}} 2(10 \mathrm{~cm})$ e $\mathrm{y}_{\mathrm{i}} 3(10 \mathrm{~cm})$, esse três últimos ao centro, ao meio e na margem da imagem), conforme já mencionado, e a avaliação de desempenho e validação desses e do modelo do protótipo laser calibrado (m.L1) foram feiras com base nos dados testes (1 e 2) de duas avaliações (aos 7 e 14 dias após transplantio, DAT). Nos testes 1 e 2 foram obtidas de 60 plantas de 20 parcelas ( 3 plantas/ parcela) as homógrafas $\mathrm{y}\left(\mathrm{d}^{\prime}\right)$ e $\mathrm{y}(\mathrm{L})$ dos parâmetros do m.L1 $\left(\mathrm{d}^{\prime}=5,944 \mathrm{~cm}\right.$ segundo calibração) e m.G $(\mathrm{L}=30 \mathrm{~cm})$ das respectivas imagens tomadas de uma altura fixa média de $100 \mathrm{~cm}$, à semelhança do observado para uma única parcela na Figura 5 (a e b). Nessa Figura 5 (C) também está indicado o processo simplificado de análise e binarização das fotografias digitais para obtenção das medidas de interesse que foram realizadas aplicando-se o Software livre ImageJ ${ }^{\circledR}$. 


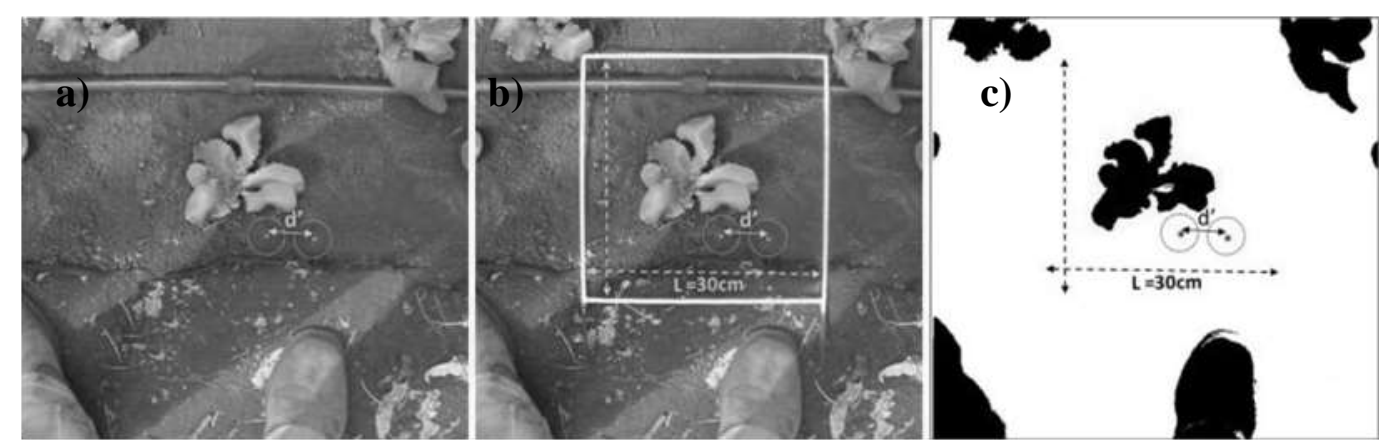

Fonte: elaborado pelo autor.

Figura 5. a) ilustração do uso e validação do protótipo laser acoplado à câmera (m.L1) para obtenção da escala (pixel $/ \mathrm{cm}$ ) e mensuração das dimensões da área de projeção da planta de alface; com uso de um par de lasers paralelo de referência d'; b) com uso de um quadrado de referência (grade) de 30 x $30 \mathrm{~cm} \mathrm{e;} \mathrm{c)} \mathrm{o} \mathrm{uso} \mathrm{dessas}$ referências na imagem binarizada e processada no software ImageJ®.

Dessa forma, utilizando-se dos dados dos testes 1 e 2 a validação e avaliação do desempenho dos modelos em comparação ao padrão (m.G) foram feitas supondo serem semelhantes às escalas obtidas das imagens por cada modelo, visto que foram tomadas com o protótipo calibrado aproximadamente da mesma distância $(\mathrm{Xf}=100 \mathrm{~cm})$. Assim, depois de obtidas as estimativas de escala da imagem (E) pelas respectivas funções com base na distância de tomada da imagem (Xf) para o m.1; na média das escalas obtidas das homógrafas (yi) do parâmetro referência (d') de todas as imagens para o m.L1, $\overline{\mathrm{E}}=$ média (Yi/d'), e na média das homógrafas (yi) do referencial (d') para os modelos m.2 e m.3, foram determinados os parâmetros de tendência e precisão dos resultados conforme Severino et al. (2004). Esses parâmetros de acurácia do equipamento foram para as medidas de precisão relativa aos erros aleatórios (dispersão ou incerteza): o intervalo de confiança ao nível de $95 \%$ de probabilidade (IC95\%) ou margem de desvio da média (MDM. 95\%) e o coeficiente de variação (CV\%). Para as medidas de tendência relativa aos erros sistemáticos (viés ou discrepância): o desvio percentual entre valor estimado $(\mathrm{E}) \mathrm{e}$ observado $(\mathrm{D} \%=100 *(\mathrm{E}-\mathrm{O}) / \mathrm{O}), \quad$ o desvio médio percentual da média (DM\%) estimada pelo modelo em relação à média observada padrão m.G (DM\%=100*(ME-MO)/MO); ou para ambos, o coeficiente de variação percentual dos desvios do modelo (Di) em relação à média do modelo padrão m.G $(\mathrm{CV} \% *$ = Desvio Padrão(Di)/MO).

\section{RESULTADOS E DISCUSSÃO}

A análise de calibração, validação e desempenho do dispositivo protótipo e modelos das funções de escala com um (m.1 e m.2) ou mais pares de laser (m.3), dependentes da distância de tomada da imagem (Xi), da homógrafa simples (Y'i) e média ( $\bar{Y}$ ) do referencial laser (d'), respectivamente, foram discutidas conforme as Figuras 6 e 7 e Tabela 1 .

Conforme Figura 6a, observou-se que a distorção variou significativamente (de forma logarítmica) até a distância de $0,4 \mathrm{~m}$ e a partir desse valor estabilizou-se com médias de distorção de $-2,8$ e 9,7 pixels para o meio e margem do seguimento médio da largura da imagem (1920/2 pixel), respectivamente, tendo sido considerada nula a distorção no centro radial da imagem. 
a)

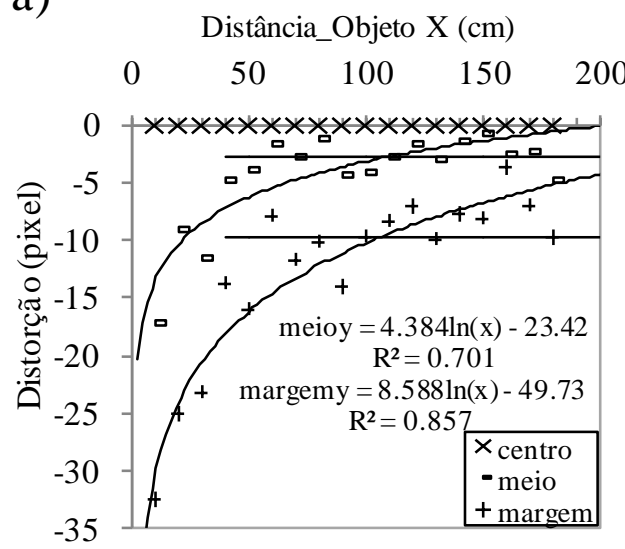

b)

$$
\text { Deslocamento radial da imagem (pixel) }
$$

$\begin{array}{llllll}0 & 200 & 400 & 600 & 800 & 1000\end{array}$

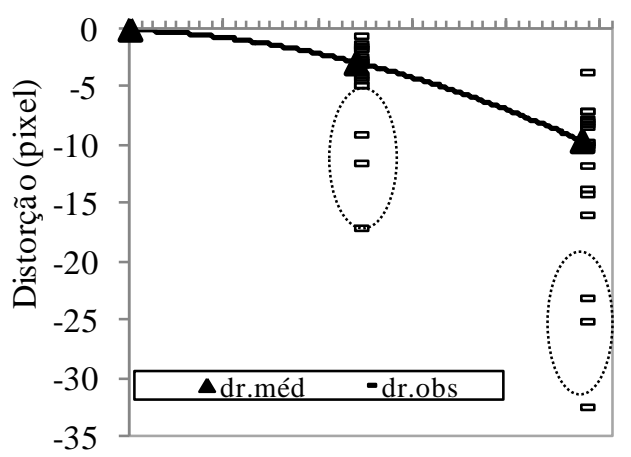

Figura 6. a) curvas de distorção do conjunto câmera-protótipo-laser com a tomada distância objeto da imagem (X) em 3 posições a partir do centro da imagem (centro, meio e margem) e; b) curva de distorção média da largura da imagem com o deslocamento radial da largura da imagem (Distância_imagem Y'i) obtida dos dados observados no intervalo $0,4 \leq \mathrm{X} \leq 1,8 \mathrm{~m}$.

Adicionalmente, observa-se ainda que a distorção variou de forma quadrática com o deslocamento radial da largura da imagem (Y'i), mais significativamente entre $-2,8$ a 9,7 pixels a partir do meio para as margens $(480<$ Y'i $<960$ pixel), ressaltando-se que essa curva média foi estimada para dados obtidos entre 0,4 e 1,8 $\mathrm{m}$ de profundidade da cena imageada (Figura 6b). Com isso, para se obter maior precisão é indicado que o objeto a ser mensurado ocupe a região central da imagem

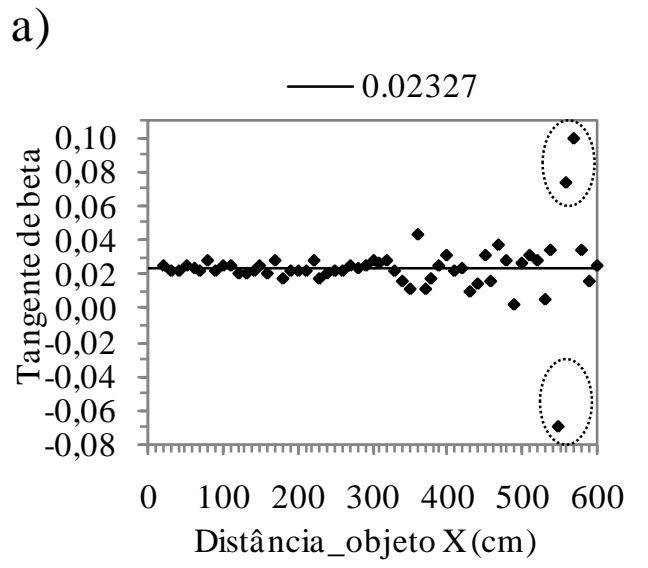

(4/16 de área) e a uma distância (profundidade) superior a $0,4 \mathrm{~m}$.

$\mathrm{Na}$ Figura 7a observa-se que os dados de tangente do semi-ângulo (beta), excluídas as extrapolações (outliers), pouco variou com a distância de tomada da imagem com média de 0,2327 , com margem de desvio médio de $\pm 0,0014$ ao nível de $95 \%$ de probabilidade e coeficiente de variação de $22,24 \%$. Com base nesse parâmetro foi possível estimar os demais ( $\beta$, Xo e d') observados (Tabelas 1 e 2 ).

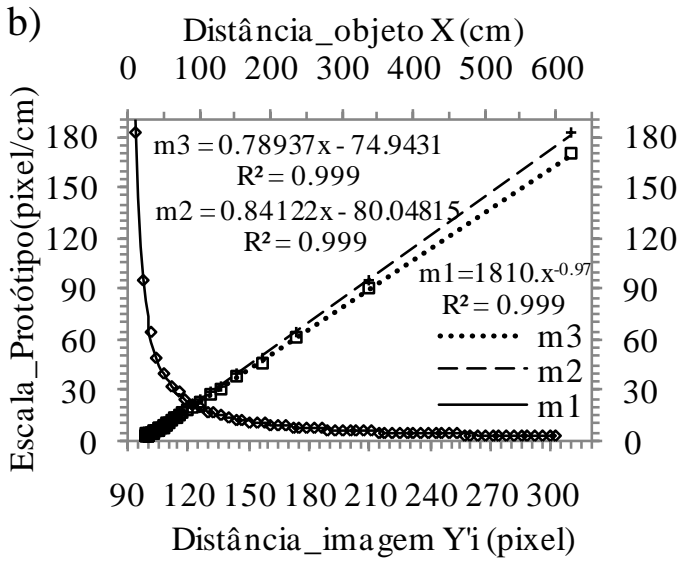

Figura 7. Tangente média obtida dos dados observados do conjunto câmera-protótipo-laser $(0,02327)$ e; Curvas dos modelos m.1, m.2 e m.3 do protótipo-laser variável com a tomada da distância objeto da imagem $(\mathrm{X})$, com a homógrafa simples (Y'i) e média ( $\bar{Y})$ do referencial laser (d'), respectivamente.

Nessa Figura 7b também são observados as funções estimadas dos modelos m.1, m.2 e $\mathrm{m} .3$, destacando-se o excelente ajuste potencial $\left(\mathrm{R}^{2}\right)$ de escala (E) com a distância de tomada da imagem (X) no m.1 e linear com a homógrafa (Y'i) do referencial (d') nos m.2 e m.3. Esses modelos de estimativa de escala foram comparados, validados e aquilatados 
segundo os parâmetros de desempenho observados para os testes 1 e 2, obtendo-se resultados semelhantes nos dois testes, conforme respectiva Tabela 1 .

Tabela 1. Parâmetros característicos do protótipo ( $\beta, \mathrm{Xf}, \mathrm{Xo}, \mathrm{d}$, e d'), escala média (E) e as medidas de desempenho (D\%, Média, Desvio Padrão, CV\%, MDM.95\%, CV\%* e DM\%) dos modelos (m.1, $2,3, \mathrm{~L} 1$ e G) em relação ao padrão (m.G), testes 1 e $2 *$

\begin{tabular}{|c|c|c|c|c|c|c|c|}
\hline \multirow[b]{3}{*}{ modelos } & \multicolumn{7}{|c|}{ Testes 1 e 2 das Avaliações 1 e $2^{*}$} \\
\hline & \multirow{2}{*}{$\begin{array}{c}\beta \\
\text { (graus) }\end{array}$} & $\mathrm{Xf}$ & Xo & $\mathrm{d}$ & $\mathrm{d}^{\prime}$ & \multirow{2}{*}{$\begin{array}{c}\mathrm{E} \\
\text { (Pixel/cm) }\end{array}$} & \multirow{2}{*}{$\begin{array}{l}\mathrm{D} \% \\
(\%)\end{array}$} \\
\hline & & \multicolumn{4}{|c|}{ 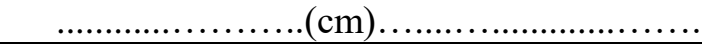 } & & \\
\hline m.1 & 1,33 & 100 & 28 & 1,3 & 5,944 & $20,78(20,78)$ & $-0,78(2,73)$ \\
\hline $\mathbf{m} .2$ & 1,33 & 100 & 28 & 1,3 & 5,944 & $\mathbf{2 1 , 0 4}(19,64)$ & $\mathbf{0 , 5 3}(-2,91)$ \\
\hline $\mathrm{m} .3$ & 1,33 & 100 & 28 & 1,3 & 5,944 & $19,92(18,60)$ & $-4,85(-8,04)$ \\
\hline m.L1 & 1,33 & 100 & 28 & 1,3 & 5,944 & $20,22(19,94)$ & $\mathbf{- 3 , 4 2}(-1,45)$ \\
\hline m.G & - & - & - & 30,0 & - & $20,93(20,23)$ & $0,00(0,00)$ \\
\hline \multirow[b]{2}{*}{ modelos } & \multicolumn{7}{|c|}{ Escala da imagem (E) } \\
\hline & $\begin{array}{c}\text { Média } \\
\text { (pixel/cm) }\end{array}$ & $\begin{array}{l}\text { DesvioP. } \\
\text { (pixel/cm) }\end{array}$ & $\begin{array}{c}\mathrm{CV} \% \\
(\%)\end{array}$ & $\begin{array}{l}\mathrm{MD} \\
(\mathrm{pi}\end{array}$ & $\begin{array}{l}.95 \% \\
1 / \mathrm{cm})\end{array}$ & $\begin{array}{c}\text { CV\%* } \\
(\%)\end{array}$ & $\begin{array}{c}\text { DM\% } \\
(\%)\end{array}$ \\
\hline m.L1 & $20,22(19,94)$ & $0,14(0,17)$ & $0,69(0,85)$ & $\pm 0,0$ & $\pm 0,04)$ & $2,52(2,31)$ & $-3,42(-1,45)$ \\
\hline m.G & $20,93(20,23)$ & $0,63(0,73)$ & $2,92(3,60)$ & $\pm 0,1$ & $\pm 0,18)$ & - & - \\
\hline
\end{tabular}

*valores entre parênteses referem-se ao teste 2 ; 1$) \beta$ - é o semi-ângulo médio do ângulo $\alpha$ formado entre os feixes do par de lasers do protótipo; 2) Xf - é a distância fixa da medida (Xi) de tomadas das imagens a partir de "Xo" no experimento com a alface; 3) Xo - é a distância fixa estimada da projeção inicial do par de lasers a partir do vértice; 4) d' - é o seguimento de desvio da projeção inicial d (formado na projeção inicial do par de lasers em Xo); 5) yi - é a medida digital da projeção (em pixel) correspondente ou homografia do seguimento referencial do modelo padrão $\mathrm{m} . \mathrm{G}(\mathrm{L}=30 \mathrm{~cm})$ ou $\mathrm{m} . \mathrm{L} 1(\mathrm{~d}$ ou d', em $\mathrm{cm})$ tomada da imagem a cada profundidade Xi

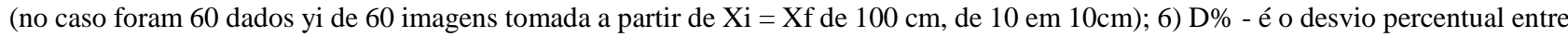
valor estimado e observado; 7) CV\% - é o coeficiente de variação dos desvios em relação à média; 8) MDM.95\% - é a margem de desvio da média ao nível de $95 \%$ de probabilidade; 9) CV\%* - é o coeficiente de variação percentual dos desvios do modelo em relação à média do modelo padrão m.G; 10) DM\% - é o desvio médio percentual da média estimada pelo modelo em relação à média observada padrão m.G.

Segundo essas análises os modelos revelaram alta acurácia, com medidas de tendência (discrepância) das estimativas muito baixa, com D\% (em valor absoluto) menor que $2,74 \%, 2,92 \%, 8,05 \%$ e $3,42 \%$ para os modelos m.1, m.2, m.3 e m.L1 tendo-se como referência o modelo padrão (m.G), destacando-se o m.2 no teste 1 e o m.L1 no teste 2 (Tabela 1). Com relação às medidas de precisão o modelo m.L1 teve alta precisão, assim como os demais modelos m.1, m.2 e m.3, tendo-se por base os baixos valores de desvio da média com CV\% de até $0,85 \%$ e MDM95\% de $\pm 0,04 \mathrm{pixel} / \mathrm{cm}$ para o m.L1 e CV\% de até 3,60\% e MDM95\% de $\pm 0,04 \mathrm{pixel} / \mathrm{cm}$ para o m.G. Ressalta-se que a maior discrepância do mod.3 entre os modelos funcionais possivelmente ocorreu em razão do grau de distorção da lente da câmera (Figura 6b).

LOPES et al., 2007, utilizaram fotos digitais para a estimativa da área foliar de meloeiro em diferentes estádios fenológicos e concluiram que o método de fotos digitais pode ser utilizado para estimar a área foliar da cultura de meloeiro, e a estimativa da área foliar de meloeiro por estádio fenológico apresenta maior precisão. ADIMI et al., 2007, avaliaram dois métodos diretos de medida de AF na cultura da soja, por meio de dimensões foliares (comprimento e largura máximos) e por imagens digitais capturadas por máquina fotográfica digital, e compararam as etimativas com o integrador foliar Li-cor, modelo LI 3100 , estes concluiram que o método de imagem digital apresenta boas estimativas de AF tanto para folhas com limbos íntegros quanto para danificados e apresenta precisão similar ao aparelho integrador de área foliar LI-COR. DALMAGO et al., 2017, avaliaram o uso de fotografias digitais para a determinação da área foliar da cultura da canola e concluiram que a área foliar da canola pode ser estimada por meio de fotografias digitais de folhas, obtidas com máquinas digitais comerciais, e que apresenta elevada acurácia em relação ao método padrão. Os 
trabalhos acima citados corroboram a utlização de fotografias digitais para a determinação de area foliar de diferentes culturas.

Assim, o desenvolvimento e uso desses dispositivos em estudos futuros poderão abrir muitas possibilidades de aplicação em pesquisas e levantamentos de dados tais como: na agricultura de precisão com o dispositivo laser sendo acoplado ao trator, veículos aéreo não tripulado (Vant ou Drone); na estimativa de fatores internos e externos da câmera visando sua calibração e modelagem específica, como é o caso da ortorretificação de imagens, e; também com potencial em pesquisas de levantamento de dados biométricos de animais silvestres de difícil acesso com o auxilio de armadilhas fotográficas entre outras muitas possibilidades de uso.

\section{CONCLUSÕES}

1) Os modelos e dispositivo protótipo desenvolvidos para estabelecer escala de imagens foram satisfatórios, e apesar de terem sido construídos e calibrados de forma simples a um baixo custo e com alta acurácia, não fazem prescindir de mais aperfeiçoamentos e estudos;

2) Os modelos e protótipos tiveram excelentes desempenhos e os parâmetros de calibração do protótipo laser e seus modelos funcionais foram estimados e ajustados adequadamente, não sendo indicado, entretanto, a utilização do dispositivo a uma distância muito próxima da câmera.

\section{AGRADECIMENTOS}

À Universidade de Brasília, Universidade Federal de Lavras, à CAPES, ao CNPq e à FAPEMIG pela disponibilização de recursos, professores e funcionários pelo apoio concedido e transferência de conhecimentos.

\section{REFERÊNCIAS}

ADAMI, M.; HASTENREITER, F. A.; FLUMIGNAN, D. L.; FARIA, R. T. Estimativa de área foliar de soja usando imagens digitais e dimensões foliares. In: Simpósio Brasileiro de Sensoriamento Remoto, XIII, 2007. Anais... Florianópolis: SBSR, 2007. p. 9-14.

ALLEN, R.G.; PEREIRA, L.S.; RAES, D.; SMITH, M. Crop evapotranspiration: guidelines for computing crop water requirements. Rome: FAO, 1998. 297 p. (FAO. Irrigation and Drainage Paper, 56).

BENINCASA, M. M. P. Análise de crescimento de plantas: noções básicas. 2. ed. Jaboticabal: FUNEP, 2003. 41 p.

BERVEGLIERI, A.; MORAES, M. V. A.; TOMMASELLI, A. M. G. Orientação e ortorretificação de imagens terrestres nadirais. Bol.Ciênc.Geod., v. 21, no 1, p.149-168, 2015.

BOSCO, L. C.; BERGAMASCHI, H.; CARDOSO, L. S.; VIVIANE AIRES DE PAULA, V. A.; CASAMALI, B. Seleção de modelos de regressão para estimar a área foliar de macieiras 'royal gala' e 'fuji suprema' sob tela antigranizo e em céu aberto. Rev. Bras. Frutic., v.34, n. 2, p. 504-514, 2012.

BRITO, J. L. N. e S.; COELHO FILHO, L. C. T. Fotogrametria digital. Rio de Janeiro: EdUERJ , 2007. 196 p.

DALMAGO, G. A.; GOUVÊA, J. A.; CUNHA, G. R.; VICARI, M. B.; KOVALESKI, S. Uso de imagens digitais para estimativa da área foliar da canola.In: SIMPÓSIO BRASILEIRO DE CANOLA, 1., 2017, Passo Fundo. Anais... Brasília, DF: Embrapa, 2017. p. 275-279.

JORGE, L. A. de C.; SILVA, D.J.C.B. AFSOFT: Manual de utilização. São Carlos: Embrapa Instrumentação, 2009. v. 1. 20p.

KAUR, G.; DIN, S.; BRAR, A. S.; SINGH, D. Scanner Image Analysis to Estimate Leaf 
Area. International Journal of Computer

Applications, v.107, n.3, 2014.

LIMA, G. S. DE; NOBRE, R. G.; GHEYI, H. R.; SOARES, L. A. DOS A.; LOURENÇO, G. DA S.; SILVA, S. S. DA. Aspectos de crescimento e produção da mamoneira irrigada com águas salinas e adubação nitrogenada. R.Bras. Eng.Agríc.Ambiental, v.18, n.6, p.615-622, 2014.

LOPES, S. J.; BRUM, B.; SANTOS, V. J.; FAGAN, E. B.; LUZ, G. L.; MEDEIROS, S. L. P. Estimativa da área foliar de meloeiro em estádios fenológicos por fotos digitais. Cienc. Rural, v. 37, n. 4, p. 1153-1156, Aug. 2007.

MARCON, M.; MARIANO, K.; BRAGA, R. A.; PAGLIS, C. M.; SCALCO, M. S.; HORGAN, G. W. Estimation of total leaf area in perennial plants using image analysis. Rer. Bras. de Eng. Agr. e Amb. v.15, n.1, p.96101, 2011.

NETO, R. P. M.; BUCK, A. L. B.; SILVA, M. N.; LINGNAU, C.; MACHADO, A. L. M.; PESCK, V. A.. Avaliação da varredura laser terrestre em diferentes distâncias da árvore para mensurar variáveis dendrométricas. Bol. Ciênc. Geod., v. 19, no 3, p.420-433, 2013.

PAVAN, N.L.; SANTOS, D. R. dos. Um método automático para registro de dados laser scanning terrestre usando superfícies planas. Bol. Ciênc. Geod., v. 21, no 3, p.572 - 589, 2015.

PEIXOTO, C. P.; CRUZ, T. V.; PEIXOTO, M. F. S. P.. Análise quantitativa do crescimento de plantas: conceitos e práticas. Enciclopédia Biosfera, v. 7, n. 13, p. 51-76, 2011.

RIOS, F. A.; SILVA, B. M.; RIBEIRO. F. C.; SILVA, W. G. DA; PETERS, P. H. C. R. Espacialização da umidade e densidade no perfil do solo cultivado com feijão-de-metro sob manejos da irrigação. Enciclopédia Biosfera, v. 9, p. 1047, 2013.

SACHET, M. R.; PENSO, G. A.; PERTILLE, R. H; GUERREZI, M. T.; CITADIN, I. Estimativa da área foliar de pessegueiro por método não-destrutivo. Ciência Rural, v.45, n.12, p.2161-2163, 2015.

SANTOS, W. F. DOS; ALVES, M. DE C.; CARVALHO, L. G. DE; RIOS, G. F. A.; FRAGA, A. C. Elaboration and Validation of a Diagrammatic Scale for Epidemiological Studies of Cercospora Leaf Spot of Castor Bean. Journal of Phytopathology, v.158, p.403 - 408, 2010.

SEVERINO, L. S.; CARDOSO, G. D.; VALE, L. S. do.; SANTOS, J. W. dos. Método para determinação da área foliar da mamoneira. Rev. Bras. de Oleag. e Fibr., v. 8, n. 1, p. 753762, 2004.

TOUMI, J.; ER-RAKI, S.; EZZAHAR, J.; KHABBA, S.; JARLAN, L.; CHEHBOUNI, A. Performance assessment of AquaCrop model for estimating evapotranspiration, soil water content and grain yield of winter wheat in Tensift Al Haouz (Morocco): Application to irrigation management. Agricultural Water Management. v.163, p.219-2351, 2016. 